\title{
LOAD-CMOD DATA ANALYSIS FOR CRACK CLOSURE
}

\author{
S.A. Seetharam and P.K. Dash \\ Structural Sciences Division, National Aeronautical Laboratory \\ Bangalore, 560017 , India \\ tel: (81)-(0812)-562179
}

A major reason for the failure of crack growth rate versus applied stress intensity factor range correlation under smoothly varying small scale yielding loadings at a given (negative) stress ratio is dissimilarities in the crack closure stress field. It is essential to assess the relevance and accuracy of crack closure measurement before coming to a conclusion that crack closure phenomenon is unable to explain the trends in growth rate in a given experiment [1]. While establishing the presence/absence of crack closure in a fatigue crack growth experiment is relatively straightforward, no general method has yet evolved to measure the effect of crack closure on the crack driving force. Load-crack mouth opening displacement (CMOD) records during fatigue cycling are routinely used for estimating crack closure. A fundamental issue is the relevance of the load level at which crack closure begins (or crack is completely open) in estimating the correct crack driving force. Elber [2] hypothesizes that crack extension does not occur while the crack is closed and considers the stress range over which the crack is fully open as the effective range for crack growth. Stress ratio effect (mainly in the positive range) and many other aspects of variable amplitude crack growth are successfully explained on this basis. Newman [3] provides an analytical justification for Elber's hypothesis by demonstrating, in a finite element simulation of fatigue crack growth, that the strain ahead of the crack tip does not vary much below the level of crack opening stress. However, in a study by Ohta et al. [4] of crack tip strains by moire fringe multiplication technique, it is shown that the strain ahead of the crack tip continues to vary well beyond the crack closure stress determined by a displacement gauge. Evidence abounds that cracks continue to grow in mode II while closed in a mode I sense in near threshold crack growth tests. On the other hand it is also reported [1] that crack growth rates are severely retarded even while the crack tip remains propped open due to asperity contact elsewhere in the wake. This discussion suggests that the load level, marking the beginning of the crack closure process (or the end of crack opening process), can not in general, be used to estimate the correct crack driving force.

There is a strong need for establishing 'benchmark' crack closure/opening stress values which lead to the correct crack driving force. These benchmark crack closure values can then be used to assess other indirect crack closure measurement techniques. In experiments where crack growth rate versus externally applied crack driving force correlation gets affected only due to crack 
closure one can determine the true crack opening stress values purely by rationalising fatigue crack growth rate data in the absence and in the presence of crack closure. This way benchmark crack closure values can be established from crack growth rate considerations. Accuracy in estimation of crack opening stress levels from experimental observation of crack closure can then be evaluated. A case study based on this approach is the subject matter of this report.

Constant stress intensity as well as constant stress amplitude fatigue crack growth tests were conducted at a stress ratio $(R)=-3$ in single edge notched specimens of BSS L-72 Al-Cu alloy of $2 \mathrm{~mm}$ thickness. A strong dependence of crack growth rate versus applied stress intensity factor range $(\mathrm{da} / \mathrm{dN}-\Delta \mathrm{K})$ correlation on stress level was demonstrated even though the stresses were well within the small scale yielding limit. The crack growth results were reported in [5]. In a similar study [6] at $R=0.7$ it was shown, by the authors, that there was no stress level effect on da/dN- $\Delta \mathrm{K}$ correlation (at low and intermediate growth rates) even at a stress exceeding the static yield strength of the material. Absence in high stress ratio tests and presence in low stress ratio tests of the effect of the applied stress level on $\mathrm{da} / \mathrm{dN}-\Delta \mathrm{K}$ correlation indicate that the effect is only through crack closure. This statement suggests that the true crack closure stress levels in these $\mathrm{R}=-3$ tests (at lower growth rate ranges) can be obtained by collapsing the $\mathrm{da} / \mathrm{dN}-\Delta \mathrm{K}$ curves onto those at a high stress ratio where crack closure is absent and static modes of failure do not affect growth rates in regime I and II of the growth rate curve. Crack closure stresses, thus derived, are termed as 'benchmark' closure stress values. Further, crack closure levels were estimated from digital load-CMOD data recorded online during the tests. A detailed discussion on crack closure study from the load-CMOD data is presented in the remainder of this report.

Typical load-CMOD data plots for the loading half cycle at different crack lengths in tests with constant $S_{\max }=40$ and $60 \mathrm{MPa}$ and constant $K_{\max }=6$ and 10 $\mathrm{MPa} / \mathrm{m}$ are shown in Fig. 1. Individual traces are numbered each corresponding to a given crack length as given in the 'insert'. It is interesting to observe that the load-CMOD responses of the closed cracks are linear and parallel to each other irrespective of crack length in the two constant stress amplitude tests. This indicates that the entire wake was closing during unloading. However, such a trend is not seen under constant $\mathrm{K}$-control tests. A sharp bilinearity is noticed in the load-CMOD response in constant stress amplitude tests, after a few millimeters of crack extension. An algorithm was developed to pick up this 'knee' point through a bilinear fit with a suitable error minimization criterion. This point was taken to represent crack opening stress level. Crack opening stress, thus obtained from load-CMOD data, is compared with the corresponding benchmark closure level in Fig. 2 for the tests discussed above. As mentioned before the benchmark closure levels were found by collapsing the $\mathrm{da} / \mathrm{dN}-\Delta \mathrm{K}$ curves onto those at $\mathrm{R}=$ 0.7 . It is interesting to observe that the trend in the opening stress variation with crack length is well described by the CMOD-estimated values. However, there are numerical differences. This discrepancy is, at least in part, due to the arbitrariness of selecting a point on the load-CMOD curve to represent opening 
stress level. In order to understand what this selected point on a load-CMOD trace means in terms of the phenomenon of crack closure, two other curves are shown in each of these plots in this figure which are discussed below.

A fourth degree polynomial was fitted to the loading half cycle load-CMOD data [7]. Over the load range representing the continuous process of crack opening, the rate of change of load with respect to CMOD (d(load)/d(CMOD) \} must continuously decrease. This property of the ideal load-CMOD response was used to estimate the stress level marking the beginning of crack opening and the stress level representing the end of the opening process. These upper and lower bound stress levels, thus obtained, are shown as the two additional curves in Fig. 2 as mentioned above. A noteworthy fact is that the 'knee' point in the load-CMOD data curve represents a load level at which the crack is still partially closed. Further the 'benchmark' closure levels are always within these bounds. This figure highlights the fact that conventional load-CMOD data analysis to obtain the stress level at which the crack is completely open can fail to estimate the correct crack driving force.

The appropriateness of the bilinear fit and the fourth degree polynomial fit to typical load-CMOD data is shown in Fig. 3a-c and Fig. 3d-f respectively. The benchmark crack opening levels are arrow marked in one set of plots (a-c). In each case (except 'f') both the bilinear as well as low order polynomial fit the experimental data well. The goodness of fit of the polynomial gives credence to the inferences drawn earlier. No single data analysis technique works uniformly well in all cases as highlighted by Fig. 3f.

The numerical differences in the benchmark crack opening and the load-CMOD-estimated crack opening stress values indicate that the stress level effect (on crack growth rate) is not totally explained through the CMOD-estimated closure. However, this partial failure (to rationalise crack growth rates obtained at a high stress ratio and at $R=-3$ with different stress levels) is due to inaccuracy in crack closure estimation. From a bilinear fit we only get an estimate of the opening stress value. The fourth degree polynomial fit to the load-CMOD data indicates that much more meaningful information regarding the crack closure phenomenon can be gleaned by suitable data analysis techniques. It is a worthwhile effort to develop algorithms to pick up benchmark crack closure values from the load-CMOD data.

Acknowledgement: This work was carried out at NAL with financial support from AR\&DB, Ministry of Defence, Government of India.

\section{REFERENCES}

[1] R.W. Hertzberg, C.H. Newton and R. Jaccard, in Mechanics of Fatigue Crack Closure, STP 982, ASTM (1988) 139-148.

[2] W. Elber, Engineering Fracture Mechanics 2 (1970) 37-45.

[3] J.C. Newman, Jr., in Cyclic Stress Strain and Plastic Deformation Aspects 
of Fatigue Crack Growth, STP 637, ASTM (1977) 56-80.

[4] A. Ohta, M. Kosuge and E. Sasaki, International Journal of Fracture 14 (1978) 251-264.

[5] S.A. Seetharam and P.K. Dash, International Journal of Fracture 52 (1991) R45-R50.

[6] S.A. Seetharam and P.K. Dash, International Journal of Fracture 52 (1991) R3-R8.

[7] K.K. Brahma, P.K. Dash and B. Dattaguru, International Journal of Fatigue 11 (1989) 37-41.

3 December 1991
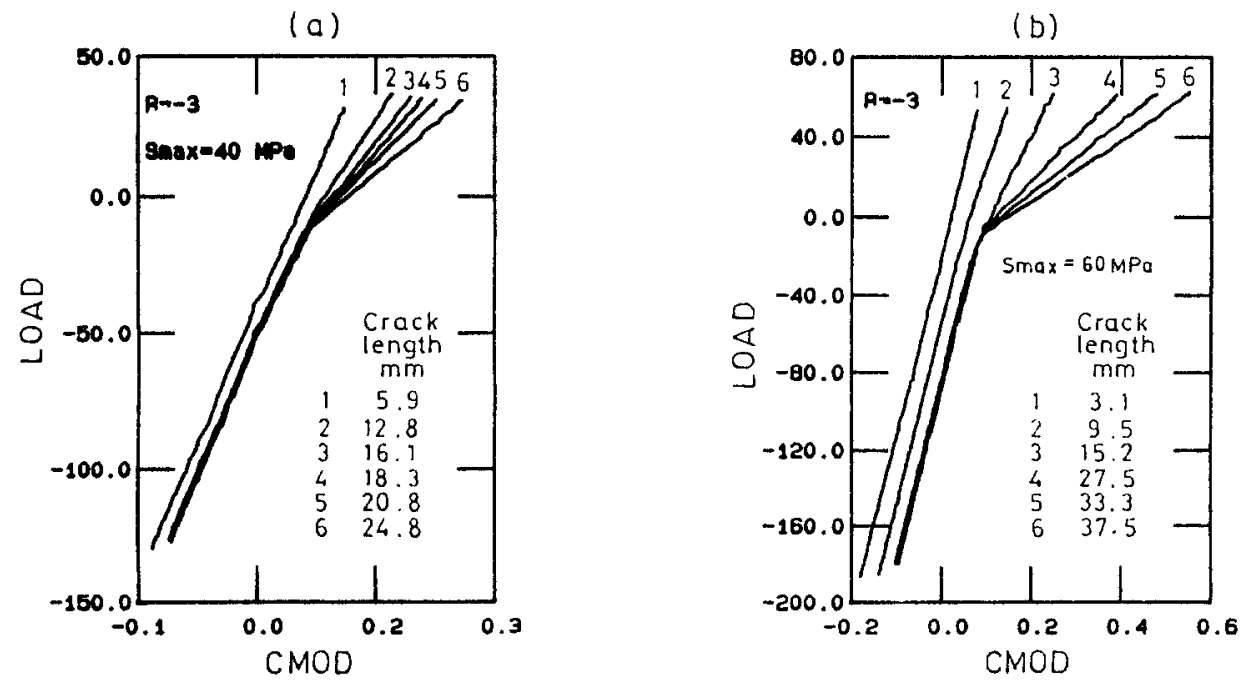

Figure 1. Load-CMOD data at various crack lengths in the loading half cycle. Shifts in the locations of load-CMOD curves in each plot are due to CMOD gage adjustments made during the course of the tests. 

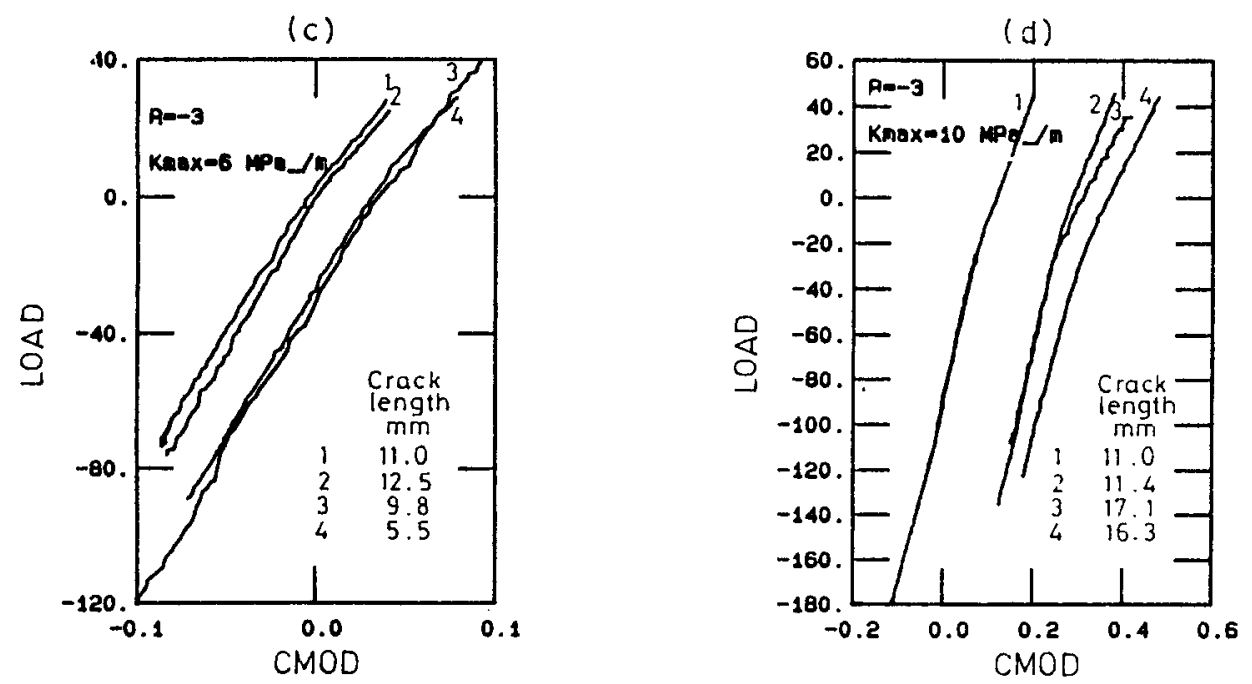

Figure 1. Continued
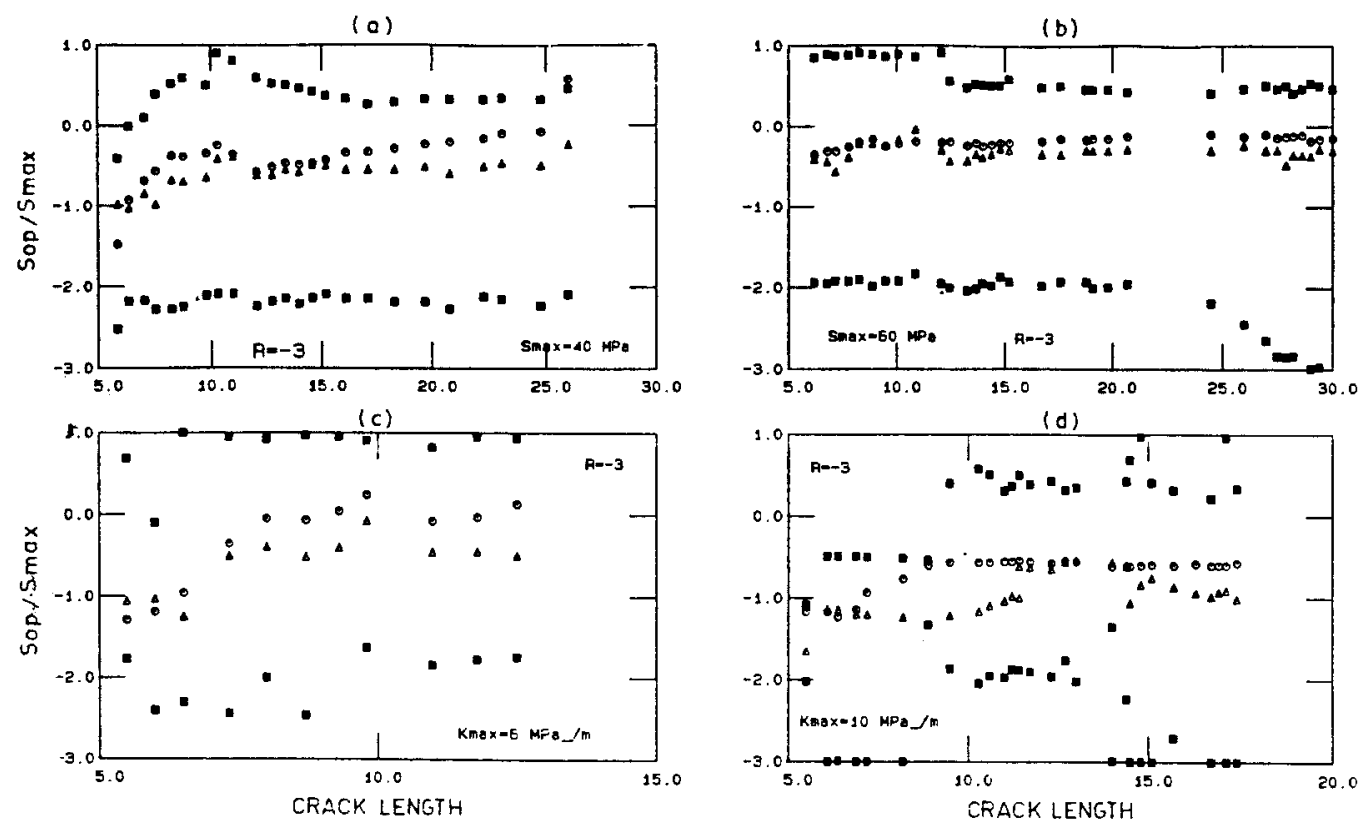

Figure 2. Crack opening stress variation with crack length. 0-benchmark, $\triangle-$ CMOD estimated, polynomial fit. 

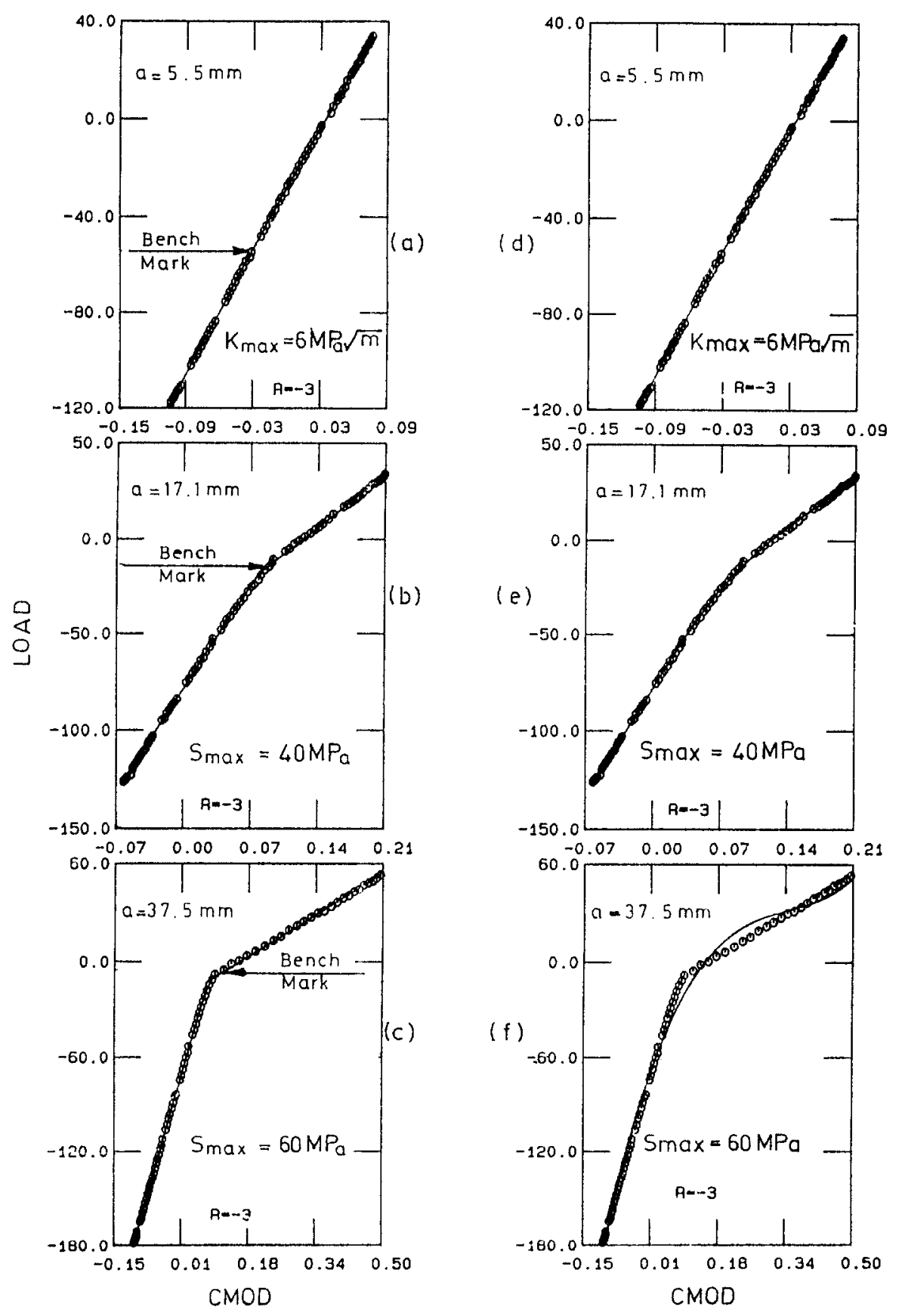

Figure 3. Analytical fit to typical load-CMOD data. Bilinear fit is shown in (a), (b) and (c). 4th degree polynomial fit for the same data is shown in (d), (e) and ( $f$ ) respectively. o-experimental data, - analytical.

Int Joum of Fracture 53 (1992) 